\title{
IV. Ueber die Bronchialdrüsentuberculose und ihre Beziehungen zur Tuberculose im Kindesalter.
}

\section{Von Dr. H. Nenmann in Berlin.}

Es ist wohl keine Krankheit gründlicher und erfolgreicher durchforscht als die Tuberculose; befruchtet durch die modernen Forschungsmethoden wächst ihr Studium ins ungemessene, und in lebhaftem Kampf der Meinungen ringen Anschauungen von grösster praktischer Wichtigkeit nach allgemeiner Anerkennung.

Im Folgenden möchte ich Einiges aus der Pathologie und Diagnose der Bronchialdrüsentuberculose hervorheben; meist alt bekannt, ist manches neuerdings mit Unrecht zurückgewiesen, manches durch die neuesten Forschungen in ein anderes Licht gerückt.

Der pathologische Anatom weiss die Wichtigkeit der Bronchialdrüsentuberculose für das Kindesalter zu würdigen. Es diene zur allgemeinen Orientirung, dass z. B. B abe s ${ }^{1}$ ) im Kinderspital zu Budapest innerhalb von 8 Jahren bei mehr als der Hälfte aller Sectionen eine Tuberculose der Lymphdrüsen, und zwar vorwiegend derjenigen des Mediastinums und der Bronchien fand. Mülle ${ }^{2}$ ) sah unter 500 Sectionen des pathologischen Instituts in München, welche die 15 ersten Lebensjahre betrafen, $126 \mathrm{mal}$ die Lymphdrüsen und hierbei 103 mal die Bronchialdrüsen tuberculös erkrankt. Anmerkungsweise führe ich an, dass ich selbst in einer fortlaufenden Reihe von 142 Sectionen, ${ }^{3}$ ) Kinder von $0-15$ Jahren betreffend, bei denen ich mein besonderes Augenmerk auf die intrathoracischen Drïsen richtete, 46 mal Bronchialdrüsentuberculose sah. Doch haben Zahlenangaben in dieser Allgemeinheit nur wenig Werth: Alter und Provenienz der Todten, vor allem aber die Beziehung zu anderen sich gleichzeitig vorfindenden Erkrankungen ermöglichen erst eine angemessene Würdigung der Bronchialdrüsenerkrankung.

Ueber die Bedeutung der Bronchialdrüsentuberculose im Bilde der Tuberculose des Kindesalters mögen folgende Zahlen eine vorläufige Vorstellung geben. Lebert ${ }^{4}$ ) sagt, dass die Bronchialdrüsen in mehr als $2 / 3$ der Fälle von Kindertuberculose tuberculös seien. Steiner und Neureutter ${ }^{5}$ ) fanden bei 302 Sectionen von Tuberculose im Kindesalter die Lymphdrüsen 299 mal und hierbei die Bronchialdrüsen 286 mal erkrankt; Fro ebeli us s ${ }^{6}$ sah die Bronchialdrüsentuberculose bei $99,2 \%$ der mit Tuberculose im St. Petersburger Findelhause verstorbenen Kinder des ersten Lebensjahres. Rilliet und Barthez ${ }^{7}$ ) sahon sie unter 312 Kindertuberculosen $249 \mathrm{mal}$; ich selbst fand unter 46 Fällen von Kindertuberculose 36 mal die Bronchialdrüsen erkrankt. Henoch ${ }^{8}$ giebt geradezu an, dass er sich in der 'That nur einzelner Ausnahmen von der Regel entsinne, dass, wenn irgendwo im Körper eines Kindes Tuberkel oder käsige Processe vorkommen, auch die Tracheal- und Bronchialdrüsen in gleicher Weise ergriffen sind.

Insoweit es sich um die pathologisch-anatomische Bedeutung der Bronchialdrüsentuberculose für das Kindesalter handelt, kann also kein Zweifel bestehen. Anders ist es mit der Aetiologie jener

1) Annales de l'Institut de Pathol. et de Bactériol. à Bucarest. I., p. 409. Bucarest 1890. 1889 .

2) Zur Kenntniss der Kindertuberculose. Munch. med. Wochenschrift

3) Dieselben wurden meist, Dank der gutigen Erlaubniss des Herrn Director Dr. P. Guttmann, im städtischen Krankhause Moabit in Berlin ausgeführt.

4) Maladies scrofuleuses et tubercul. Paris 1849

5) Prager Vierteljahrsschrift 1865 II, p. 34.

6) Jahrb. f. Kinderheilkunde Bd. 24, p. 71.

i) Handb. d. Kinderkrankh. Uebersetzt von Hagen. 1855

8) Vorlesungen tiber Kinderkrankh. IV. A ufl., p. 413. 
Erkrankung und der sich hieraus ergebenden Bedeutung der Bronchialdrüsentuberculose für eine gleichzeitige Tuberculose anderer Organe.

Hier müssen wir zunächst die viel umstrittenen Bezieh ungen zwischen Scrophulose und Tuberculose beleuchten. Ohne auch nur einen flüchtigen Blick auf die endlose Reihe von anatomischen und klinischen Arbeiten zu werfen, welche sich mit dieser Frage beschäftigt haben, errinnere ich nur daran, dass nach der Virchow'schen Lehre die Scrophulose in der grossen Hinfälligkeit der entzündlichen Producte ınd ihrer hierdurch veranlassten Neigung zư Verkäsung begründet war; in den verkästen scrophulösen Drüsen entwickelte sich dann mit Vorliebe als eine heterogene Geschwulstbildung der Tuberkel. Die Versehiedenartigkeit von Scrophulose und Tuberculose wird noch jetzt von ersten Autoritäten anatomisch und klinisch aufrecht erhalten. Zwar hat schon vor einer Reihe von. Jahren die histologische Untersuchung die Verwandtschaft tuberculöser und scrophulöser Processe nachgewiesen, zwar zeigte das Experiment, dass gerade der Käse scrophulöser Organe zur experimentellen Erzeugung der Tuberculose besonders geeignet sei, aber erst nach Entdeckung des Tuberkelbacillus durfte man hoffen, der Unität von Scrophulose und Tuberculose allmählich Anerkennung zu verschaffen. Bekanntlich wies Koch seinen Bacillus in einer Reihe von scrophulösen Processen nach, und dieser Nachweis wurde bald darauf in emsiger Arbeit von zahIreichen Autoren ergänzt; er wurde für den Lupus, die scrophullösen Drïsen, die fungösen und cariösen Processe, die kalten Abscesse, selbst bei einzelnen Ekzemen geführt, und zwar von so verschiedenen Seiten, dass es erübrigt, auf die einzelnen Untersuchungen hier einzugehen. Freilich vermochte dieser Nachweis nicht den Unitariern zu einem bedingungslosen Sieg zu verhelfen; denn es war schliesslich mit dem Bacillennachweis nicht mehr als das Auftreten echter Tuberculose in den scrophulösen Organen - eine altbekannte Thatsache - in neuer Form bewiesen. Gegen die ätiologische Identität beider Processe wendete man ein, dass sich der Bacillus nicht regelmässig und zudem zu spärlich finde, um „die oft gewaltig entwickelten Leiden zu erklären, den klinischen Verlauf solcher multipler scrophulöser Knochen- etc.Leiden noch weniger ${ }^{6 *}$ (Biedert) ${ }^{1}$ ). Würde der scrophulöse Process spontan ausheilen können, wenn er eine wahre Tuberculose wäre, und müsste man nicht in der Lage sein, das Bild der Scrophulose ebenso wie das der Tuberculose experimentell durch den Tuberkelbacillus zu erzeugen? Hieraıf wäre zu erwiedern: zunächst ist der Tuberkelbacillenbefund in den scrophulösen Krankheiten sehr constant; mikroskopisch ist freilich der Bacillus vielfach nur nach langem Suchen und nur in vereinzelten Exemplaren aufzufinden, wovon, nebenbei bemerkt, auch Ausnahmen vorkommen (es finden sich z. B. in dem erweichten Käse scrophulöser Bronchialdrüsen unter Umständen die Bacillen in ungeheurer Menge vor). Doch kann sich der Bacillus noch in den scrophulösen Organen selbst finden, wenn sein mikroskopischer Nachweis in dem von ihnen producirten sogenannten Eiter nicht mehr gelingt. Nimmt man die Verimpfung auf empfängliche Thiere zu Hülfe, so ist er freilich auch noch in letzterem durch die Experimentaltuberculose nachweisbar. Ein kleiner Procentsatz von Fehlimpfungen wird hiergegen nichts beweisen, wenn man mit der Möglichkeit von Versuchsfehlern oder der Anwendung ungeeigneten oder nicht ausreichenden Materials rechnet..2)

Wenn nun Scrophulose und Tuberculose sich zu einander nicht anders verhalten sollen „als der Boden, auf dem eine Pflanze mit Vorliebe wächst, zu dieser Pflanze selbst" (Kanzler ${ }^{3}$ ), so trifft diese Auffassung. eben deshalb nicht $\mathrm{zu}$, weil die Pflanze hier nicht mit Vorliebe, sondern ausschliesslich auf diesem Boden wächst, und ausserdem das, was Kanzler und andere für den Boden halten, bei genauerem Zusehen einen Theil der Pflanze selbst darstellt. Der Tuberkelbacillus findet sich so gut wie in jedem Falle und in jedem Stadium anatomischer Scrophulose, selbst dann, wenn maskroskopich noch keine Verkäsung vorhanden ist, ${ }^{4}$ ) und es stehen die beiden coexistirenden Processe so nahe, dass es schwer hält zu sagen, wo die Scrophulose aufhört und die Tuberculose anfängt. Von entscheidender Bedeutung ist aber in dieser Streitfrage das pathogenetische Verhalten der Tuberkelbácillen.

Es hängt der Verlauf einer Infection mit dem Bacillus von drei Momenten ab: von der Zahl der eingeführten Bacillen, von ihrer Virulenz, von der Empfänglichkeit des inficirten Thieres; letztere lässt sich wieder in eine locale und allgemeine Disposition

1) Vgl. Biedert, Lehrb. d. Kinderkrankh. IX. Aufl. 1887, p. 536.

2) Man sollte z. B. die negativen Versuche Gi esler's jetzt nicht mehr heranziehen, welche auf Grund der späteren Erfahrungen ohne weiteres als fehlerhaft betrachtet werden dürfen.

3) Berlin. klin. Woch. 1884.

4) Z. B. erzeugte ich durch Verimpfung eines einfach hyperplastischen scrophulösen Lymphoms, welches aus der Halsgegend exstirpirt war, beim Meerschweinchen eine in 72 Tagen tödtlich verlaufende Impftuberculose. gliedern. Die Bedeutung des ersten Punktes wies Wyssokowit sch ${ }^{1}$ ) ïberzeugend im Experiment nach, in Uebereinstimmung mit den Befunden Garrè's und den Anschaungen Baumgarten's ${ }^{2}$ ), dass auch beim Mensehen die Infectiosität tuberculösen Eiters seinem Gehalt an Tuberkelbacillen entspricht. Ebenso wie die geringe Anzahl von Bacillen kann aber auch eine herabgesetzte Virulenz dazu führen, dass es nur zu einer localen Erkrankung kommt, welche langsam verläuft und unter Umständen ausheilt. (Vergl. Löte ${ }^{3}$ ). Besonders möchte ich hierbei auf die Versuche von Courmont und Dor ${ }^{4}$ ) verweisen, welche lange ausserhalb des menschlichen Körpers cultivirte Tuberkelbacillen in die Blutbahn junger Kaninchen injicirten: gegen den 6. Monat fingen diese an abzumagern, und es entwickelten sich als einzige Localisation der Tuberculose tuberculöse Gelenkerkrankungen, welche dem Tumor albus beim Menschen entsprachen. Als drittes Moment käme die wechselnde Empfänglichkeit in Betracht, wie sie für die verschiedenen Thierspecies (z. B. Meerschweinchen, Kaninchen, Hunde, Rindvieh) bekannt ist; gleichzeitig findet sich hier eine Verschiedenheit in der Entwickelung des tuberculösen Processes: bei starker Disposition eine rapide Entwickelung miliarer Tuberculose, welche zum Tode führt, bevor noch makroskopisch eine Verkäsung zu bemerken ist; bei geringerer Disposition die Bildung localer Heerde mit Neigung zu ausgedehnter Nekrose des neugebildeten Gewebes, welche bald zur Verkäsung, bald zur Erweichung, zur Verkalkung u. s. w. führt. Abgesehen hiervon wird aber der Bacillus dieselbe Species je nach der Art der Einverleibung verschieden schnell und intensiv inficiren; es macht einen Unterschied, ob wir ihn z. B. in eine Vene oder in ein Gelenk einführen; in dem einen Fall überschwemmt er momentan den ganzen Organismus, in dem anderen bleibt er zunächst in einem wenig disponirten Organ eingeschlossen.

Man kann hiernach kaum bezweifeln, dass der Tuberkelbacillus eine scrophulöse Erkrankung hervorzurufen befähigt ist. Es könnte sich nur darum handeln festzustellen, welche Momente hierbei den Ausschlag geben. Arloing ${ }^{5}$ ) glaubte experimentell beweisen zu können, dass die Bacillen in den scrophulösen Heerden weniger virulent wären; doch konnte man seine Resultate vielleicht auch durch die geringe Menge der verimpften Bacillen erklären, wie sie denn überhaupt nicht unwidersprochen blieben (de Renzi. ${ }^{6}$ ) Zudem spricht direkt gegen eine herabgesetzte Virulenz der in den scrophulösen Producten eingeschlossenen Bacillen die Häufigkeit, mit der eine maligne Tuberculose von ihnen ausgeht. Dass bei der Scrophulose die Bacillen einen nur localen tuberculösen Process elzeugen, bei dem die Neubildung tuberculösen Gewebes gegenüber der Necrobiose zurücktritt, dass hier mit dem Tod der Gewebe auch die Bacillen immer wieder bis auf einen kleinen Rest ihren Untergang finden dies spricht freilich für eine wenig energische Activität des Tuberkelbacillus, aber es dïrfte für ihre Erklärung einerseits die jeweilige, für die Generalisation ungünstige Localisation des Processes ausreichen, andererseits der zeitweise Mangel einer stärkeren Disposition für die tuberculöse Infection heranzuziehen sein. Es erscheint hierbei übrigens zweifelhaft, ob sich der kindliche Organismus von der Localisation der Infection abgesehen - wesentlich von dem erwachsenen verschieden zeigt, nachdem man im Gegensatz zu früheren Anschauungen auch bei letzterem das häufige Vorkommen höchst chronischer und selbst zur Heilung führender tuberculöser Erkrankungen kennen gelernt hat.

Ist insofern auch der Einwand des Klinikers, dass Serophulose im Gegensatz zur Tuberculose chronisch verlaufen und sogar heilen könne, heute nicht mehr stichhaltig, so ist der Kliniker doch durch den Nachweis, dass anatomisch als scrophulös bezeichnete Veränderungen eine tuberculöse Basis haben, noch nicht ohne weiteres gezwungen, den Begriff der Scrophulose aufzugeben. Man zieht klinisch den Kreis der scrophulösen Erkrankungen viel weiter als anatomisch. Sondern wir also von den specifisch scrophulösen Processen zunächst die nachweisbar tuberculösen Affectionen ab, als da sind: tuberculöse Lymphome, Knochencaries - mag sie in den Epiphysen oder als Spina ventosa in den Diaphysen auftreten, mạg sie die grossen Röhrenknochen, die kleinen Knochen der Hand und des Fusses, die Wirbelsäule oder die Ohrknochen betreffen ferner fungöse Gelenkentzündungen, tuberculöse Abscesse des Unterhautgewebes (Scrophuloderma), tuberculöse Senkungsabscesse, Lupus, tuberculöse Geschwüre der Haut und Schleimhäute (mit dem Sitz in Conjunctiva, Nase, Rachen, Scheide ete.). Nach Abzug aller dieser Affectionen bleibt noch, nach einigen klinischen Autoren zu

1) Verhandlung des X. internat. Congresses. II. Bd., p. 180.

2) Jahresbericht II, 1886, p. 233

${ }^{3)}$ Ein Beitr. z. experiment. Pathol. d. Tuberc. Refer. Arch. f: Kinderk. XII, p. 128 .

4) Compt. rend. de la soc. de Biologie 1890 , p. 587.

5) Le Progrès méd. 1886 No. 41.

6) Vergl. Baumgarten's Jahresbericht III, p. 210. 
schliessen, ein buntes Bild der verschiedensten scrophulösen Erkrankungen. Zwar behandeln wir nicht mehr, wie es Hufeland ${ }^{1}$ ) in seinem mit Recht berühmten Werke that, in diesem Kapitel den Cretinismus und die Taubstummheit, aber es finden sich doch hier auch heute noch, nach Aussonderung der an den gleichen Organen auftretenden tuberculösen Affectionen, eine Anzahl Organerkran kungen angeführt, die vielleicht nicht mit grösserem Recht den tcrophulösen Krankheiten zugerechnet werden dürfen. Wenn es z. B. in dem Kapitel "Serophulose" heisst: "Blennorrhoische Affecsionen der Vaginalschleimhaut kommen insbesondere bei grösseren Mädchen vor"'2), so können doch hier, nach Abzug der sehr seltenen tuberculösen Erkrankungen der Vagina (vergl. einen Fall von Demme), wesentlich nur durch mechanische Reizung erzeugte Katarrhe sowie specifisch gonorrhoische Eiterungen gemeint sein, bei denen ich ebenso wenig wie andere Autoren den Gonorrhoecoccus je vermisste. Wird man, wenn man das Scrophuloderma abgesondert hat, die multiplen Abscesse im Unterhautgewebe der Säuglinge mit ihrem acuten, wenngleich recidivirenden Verlauf und constanten Befund von Eitercoccen heute noch zur Scrophulose rechnen dürfen? Oder welches Anrecht hat die bei Kindern so häufige Entzündung des Mittelohrs - nachdem die tuberculöse Otitis in Abzug gebracht ist -, mit der Scrophulose in Beziehung gebracht zu werden, da sie fast immer im Gefolge von Infectionskrankheiten oder nicht specifischen Katarrhen des Rachens durch Fortleitung des Entzündungsprocesses entsteht? Ebenso wenig ist eine chronisch-katarrhalische oder eitrige Rhinitis mit dem consecutiven Ekzem des Naseneinganges und der chronischen Verdickung der Oberlippe durch Verlauf oder Reaction auf therapeutische Eingriffe an und fitr sich als scrophulös charakterisirt, wofern dies nicht von Fall zu Fall durch besondere, wohl tuberculöse Veränderungen (Perichondritis resp. Periostitis der Nasenwurzelknochen) nachgewiesen ist ${ }^{3}$ ). Nächst den Schleimhäuten soll der bevorzugteste Tummelplatz der Scrophulose die Haut sein: die freilich nur kleine Zahl von Ekzemen und Hautulcerationen, in denen der Tuberkelbacillus nachgewiesen werden konnte (Falle von Demme und Volkmann), wird man nicht als scrophulös beanstanden; wenn aber die ganze übrige Schaar der bei Kindern so häufigen Hauterkrankungen, von der Impetigo contagiosa und dem impetiginösen Ekzem bis zum Strophulus und der Prurigo, für die Scrophulose beansprucht wird, so muss doch auch hier die Ansicht das Uebergewicht gewinnen, dass bald allgemeine Verhältnisse im Bau des kindlichen Hautorgans, bald locale Umstände, wie vermehrte Schweiss- oder Talgbildung, bald bestimmte Zustände anderer Organe, wie z. B. des Verdauungscanals, im Spiele sind (vergl. Pins (l. c.) und Bohn $)^{4}$, sich aber keinenfalls alle die verschiedenen Erkrankungen auf die Scrophulose als, letzte Ursache zurtickfithren lassen. Und ebenso ist es schliesslich auch mit dem Lymphdrïsensystem; auf der einen Seite tuberculöse Erkrankungen, auf der anderen chronische ohne Besonderheit, durch andauernde oder recidivirende Reize verschiedenster Art, welche aus dem Wurzelgebiete zugeführt werden, veranlasst; ganz zu schweigen von solchen z. B. von $\mathrm{Rabl}^{5}$ ) als scrophulös aufgefassten .Lymphdrisenschwellungen, die nicht verkäsen, sondern nach einiger Zeit wieder verschwinden, oder solchen, die unter der Erscheinung einer gewöhnlichen acuten Entzündung mit Eiterung rasch verlaufen." (Fortsetzung folgt.)

1) Ueber die Natur, Erkenntniss und Heilart der Scrophelkrankheit. 3. Aufl. Berlin 1819 .

2) Unger, Lehrbuch der Kinderkrankheiten. Leipzig und Wien 1890. p. 448 .

3) Vergl. Pins, Ueber die Beziehung der Scrophulose zu einzelnen Symptomen derselben. Archiv f. Kinderheilk. $X$.

$\left.{ }^{4}\right)$ Jahrb. f. Kinderheilk. N. F. XX, p. 45.

5) Jahrb. f. Kinderheilk. 1888. N. F. XXVII. 